УДК: 539.22

\title{
ФОРМИРОВАНИЕ САМООРГАНИЗОВАННЫХ И ПЛАНАРНЫХ СТРУКТУР В МИКРОПЛАЗМЕ ИСКРОВОГО РАЗРЯДА
}

\author{
(ㄷ) 2018 И. И. Долгих, Д. В. Авдеев, Т. В. Куликова, Л. А. Битюцкая \\ Воронежский государственный университет, Университетская пл., 1, 394018 Воронеж, Россия \\ e-mail:dolgihigor@yahoo.com
}

Поступила в редакцию 24.04.2018

\begin{abstract}
Аннотация. Изучены особенности взаимодействия слоистых прекурсоров с микроплазмой искрового разряда. Рассмотрены механизмы формирования самоорганизованных микрочастиц и влияние природы исходных материалов на форму частиц.
\end{abstract}

Ключевые слова: микроплазма, микрочастицы, слоистые прекурсоры, искровой разряд, самоорганизация.

DOI: https://doi.org/10.17308/kcmf.2018.20/525

\section{ВВЕДЕНИЕ}

В настоящее время разработано достаточно большое количество разнообразных методов получения графена и других 2D материалов, начиная от механического расслаивания слоистых прекурсоров и заканчивая сложными и дорогостоящими методиками послойного эпитаксиального выращивания в случае, если исходные компоненты не образуют слоистых аллотропов. Для слоистых материалов можно выделить три основные применяемые методики: микромеханическое и жидкофазное расслоение и Ван-дер-Ваальсова эпитаксия [1-3]. При этом известный метод микромеханического расслоения позволяет получать двумерный материал высокого качества, но непригоден для промышленного производства. Увеличить выход материала позволяет метод жидкофазного расслоения, недостатком которого является необходимость исключения негативного влияния среды протекания процесса, например, окисление материала. Ван-дер-Ваальсова эпитаксия позволяет получать качественные листы материала правильной геометрической формы, но на подложках определенного типа - с отсутствием оборванных связей на поверхности.

Ранее нами была показана возможность получения планарных структур, структур типа «ядрооболочка» и композитов на основе сурьмы различными методами: как термическими, так и методом жидкофазного расслоения прекурсора [4-6].
Один из многообещающих методов получения двумерных материалов - это получение в искровом разряде. Он позволяет точно контролировать размеры частиц и чистоту материала, а также является экологически чистым, поскольку весь процесс синтеза происходит в закрытом объеме и не требует притока и оттока реактивов [7].

Производительность метода определяется количеством материала электрода, испаряемого за один импульс, и частоты следования импульсов. Для повышения производительности в реакционной зоне располагают множество одинаковых разрядников и продувают их потоком газа, уносящего уже образовавшиеся частицы. В этом случае производительность зависит от мощности питающей установки и скорости потока газа [8].

В распространенных методах частицы размером 5-10 нм образуются в зоне разряда и уносятся потоком газа в другую камеру, где уже они собираются в агломераты размером в 100 нм, имеющие произвольную форму [9].

Данный метод, разработанный в 1980 г. для получения аэрозолей наночастиц, применялся к разным материалам и комбинациям материалов, позволяя производить микролегирование. Получались слипшиеся частицы неправильной формы с хорошо контролируемым составом. При этом размеры изначальных не агрегированных частиц зависят от материала. Используя электроды из двух 
чистых материалов, получают наночастицы сплава, благодаря высокой температуре искры, намного превышающей температуры кипения используемых материалов [10].

Искровой разряд в воздухе при нормальных условиях состоит из узких токопроводящих каналов, распространяющихся с большой скоростью и поддерживаемых фотоионизацией воздуха. Диаметр стримера пропорционален корню из его длины, в разрезе он представляет собой эллипсоид вращения. Скорость распространения стримера пропорциональна его длине, а также отношению электрического поля к давлению газа. Области усиленного поля концентрируются на концах стримера. Поверхность стримера неустойчива и должна существовать $10^{-9}$ с, однако существует $10^{-7}$ с, поскольку неустойчивость временно подавляется [11].

В канале слаботочной искры возникают токовые пульсации продолжительностью в десятки нс, разделенные промежутками в десятки микросекунд, а рост тока сопровождается сжатием искрового канала. При увеличении приложенного напряжения ток растет за счет учащения импульсов [12]. Пульсации также наблюдались и в других типах газовых разрядов [13].

Образование частиц в искровом разряде происходит в две стадии. В начальный момент времени, когда температура разряда высока и высоко сращивание частиц, успевают образоваться маленькие частицы сферической формы. Затем температура в разряде резко падает, и далее эти частицы соединяются в крупные частицы произвольной формы. Образовавшиеся на начальной стадии частицы растут за счет захвата атомов из паров и за счет столкновений. Если преобладает первое - то получаются сферы, если второе - то дендроподобные агломераты. Первоначальные частицы имеют размеры от 1 до 10 нм в зависимости от материала и параметров разряда и формируются за сотни микросекунд [14], т. е. за время существования единичного искрового канала, и процессы в следующем канале можно считать независимыми. Согласно распространенной гипотезе крупные сферические частицы - это капли, оторвавшиеся от кончика электрода в результате отдачи после удара плазмой, поэтому для борьбы с ними электрод охлаждают [14].

В существующих искровых генераторах отрицательные частицы преобладают, предположительно, из-за осаждения положительных на заземленном корпусе [14]. Для частиц $\mathrm{Al}, \mathrm{C}$ и $\mathrm{Cu}$ исследовалась доля нейтральных частиц. Частицы менее 10 нм практически нейтральны, при увеличении размеров до 50 нм заряженных частиц становится половина [15].

Плазма, возникающая благодаря электрическому полю - неравновесная. Температура ионов и нейтральных частиц намного уступает энергиям электронов. Температура газа определяется конкуренцией двух процессов - передачи энергии от электронов ионам и нейтральным частицам и охлаждением последних частицами окружающей среды. Если преобладает первый процесс, то температура газа может быть очень высокой (20000 К), если же преобладает второй процесс, то низкой $\left(50{ }^{\circ} \mathrm{C}\right)[10,16]$. Низкотемпературная плазма позволяет работать с материалами, не выдерживающими высоких температур. Пространственное ограничение плазмы приводит к росту плотности и температуры электронов [17].

В разряде на воздухе при напряжении в несколько кВ в присутствии паров воды образуются радикалы и ионы, быстро расходующиеся во взаимодействиях между собой. Также образуются озон и перекись водорода. Разряд над водой понижает значение $\mathrm{pH}$, в воде накапливаются окислители и восстановители. Горячий канал разряда испускает фотоны УФ диапазона [18].

Из-за резкого перепада температуры при потухании искры образуются перенасыщенные пары, которые конденсируются во фрактальные структуры [19].

Искровой метод является универсальным, в монографии [15] систематизировано его применение как для тугоплавких материалов с низким давлением паров (W, Mb), так и для легкоплавких с высоким давлением паров ( $\mathrm{Sb}, \mathrm{Mg})$. Метод может применяться как лабораторный и как промышленный. Влияние анизотропии материалов на результат обработки в литературе не освящен. Изучено только влияние природы материала на выход продукта.

Цель работы - анализ физико-химических процессов и оптимизация режимов получения планарных структур при электроимпульсной обработке слоистых прекурсоров с ковалентным межслойным взаимодействием.

\section{МАТЕРИАЛЫ И МЕТОДЫ}

Для импульсного воздействия плазмой на слоистый материал использовался искровой разряд. Для исключения плавления и окисления использовали холодную плазму барьерного разряда. Разряд производился в сухом воздухе при нормальных условиях между электродами, выполненными из исследуемых материалов. В качестве диэлект- 
рика для получения барьерного разряда использовалась слюда и ситалл. Импульсы напряжением в 20 кВ и продолжительностью 10 мкс получали по схеме катушки Румкорфа. Форма импульсов контролировалась осциллографом с помощью емкостного датчика (рис. 1). Образующиеся в разряде частицы оседали на скотч, расположенный в пространстве под электродами.

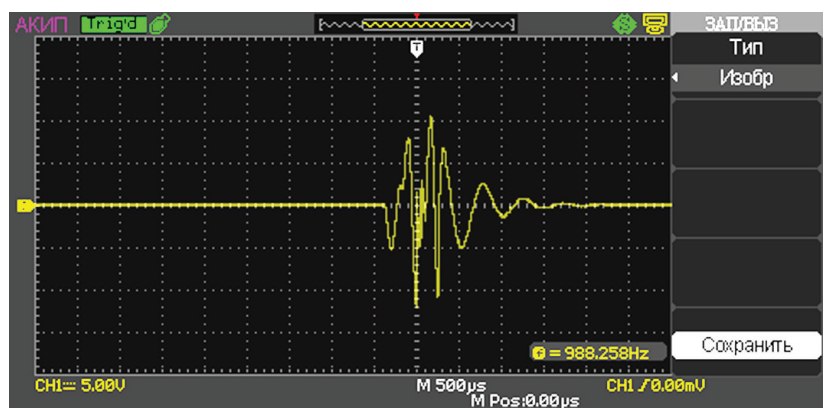

Рис. 1. Осциллограмма подаваемого на электроды импульса, снятая с помощью емкостного датчика

[Fig. 1. The oscillogram of the pulse applied to the electrodes, measured with a capacitive sensor]

В качестве исходных материалов использовались монокристаллические $\mathrm{SiC}$ и InSb, а также поликристаллические $\mathrm{Sb}$ и $\mathrm{Bi}$.

Морфология полученных частиц исследовалась с помощью растровой электронной микроскопии. Состав контролировался с помощью энергодисперсионного анализа.

\section{РЕЗУЛЬТАТЫ ЭКСПЕРИМЕНТА}

В результате электроимпульсного воздействия на электроды наблюдалось образование структур различных морфологий: сферы, фракталы и планарные структуры. Если образование сфер и фракталов обусловлено термическими процессами, описанными в литературе, то образование планарных структур (рис. 3) свидетельствует о процессе нетермического расслоения материала за счет разрыва межслоевых ковалентных связей под воздействием сильного импульсного поля. Образование сфер обусловлено поверхностным плавлением с последующим отрывом капли (рис. 2). Фрактальные структуры вырастали на поверхности исходных электродов в результате конденсации заряженных паров (рис. 4). Соотношение тепловых и полевых эффектов определялось степенью анизотропии обрабатываемого материала и его температурой плавления, а также температурой плазмы разряда, поэтому его можно контролировать. Сурьма производила сферы от 1 до 10 мкм из-за низкой температуры плавления, фракталы изза высокого давления насыщенных паров и слои

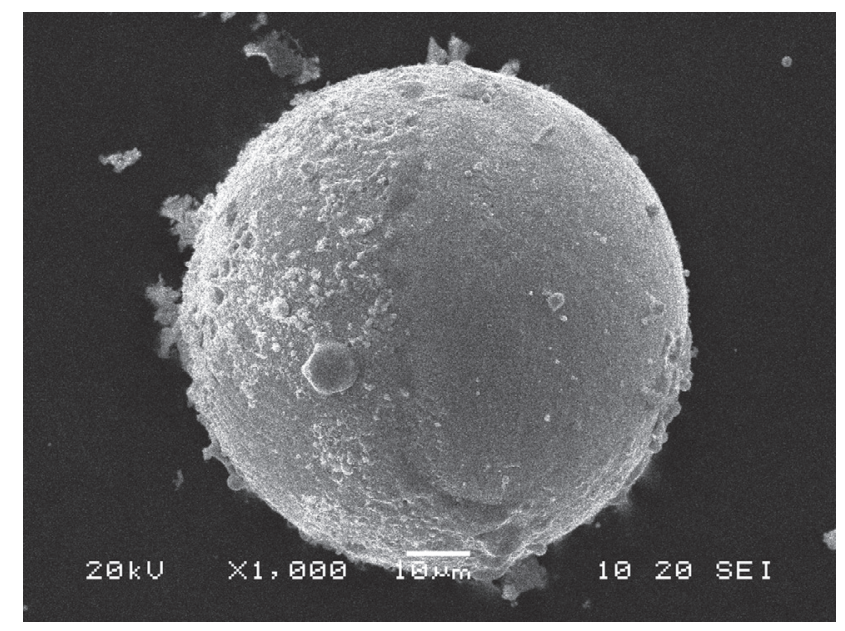

$a$

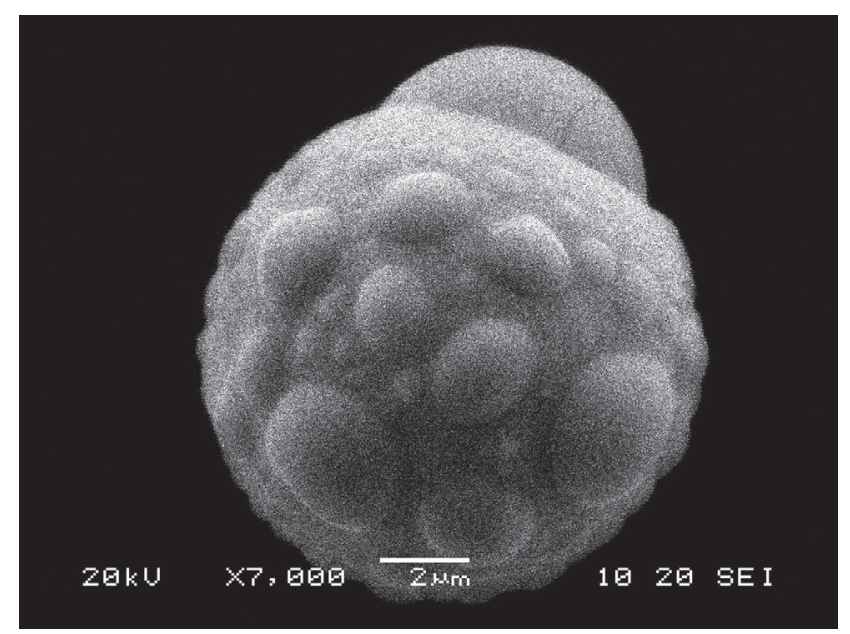

$b$

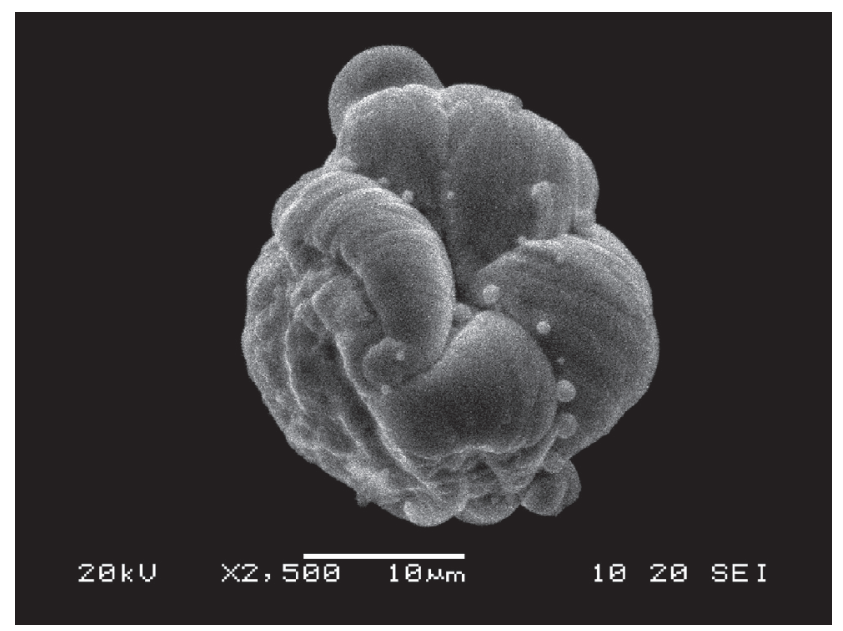

c

Рис. 2. СЭМ изображения частиц, образованных в результате поверхностного плавления материала электрода: $a-\mathrm{Sb}, \times 1000,20$ кB; $b-\mathrm{Bi}, \times 7000,20$ кВ; $c-\mathrm{InSb}, \times 2500,20 \mathrm{\kappa B}$

[Fig. 2. SEM images of particles formed as a result of surface melting of the electrode material $a-\mathrm{Sb}, \times 1000$, $20 \mathrm{kV} ; b-\mathrm{Bi}, \times 7000,20 \mathrm{kV} ; c-\mathrm{InSb}, \times 2500,20 \mathrm{kV}]$ 

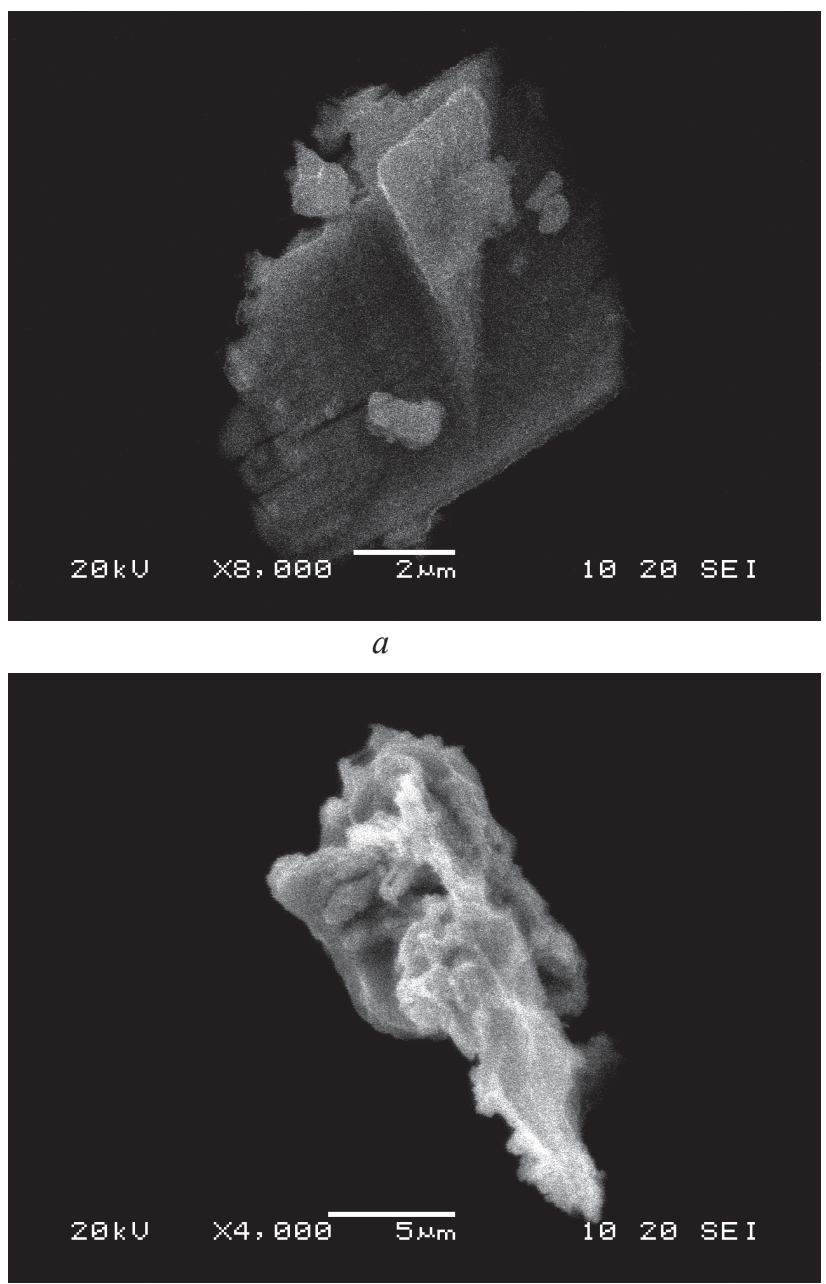

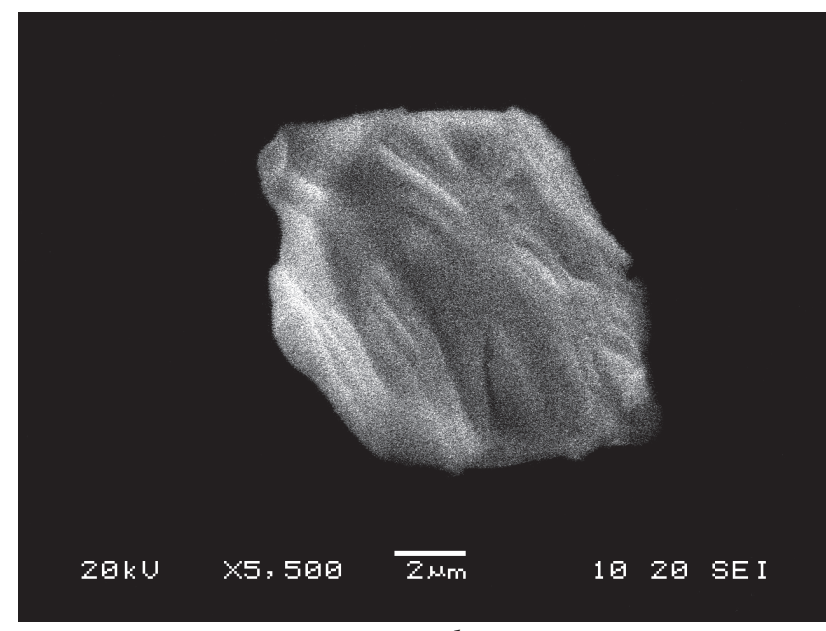

$b$

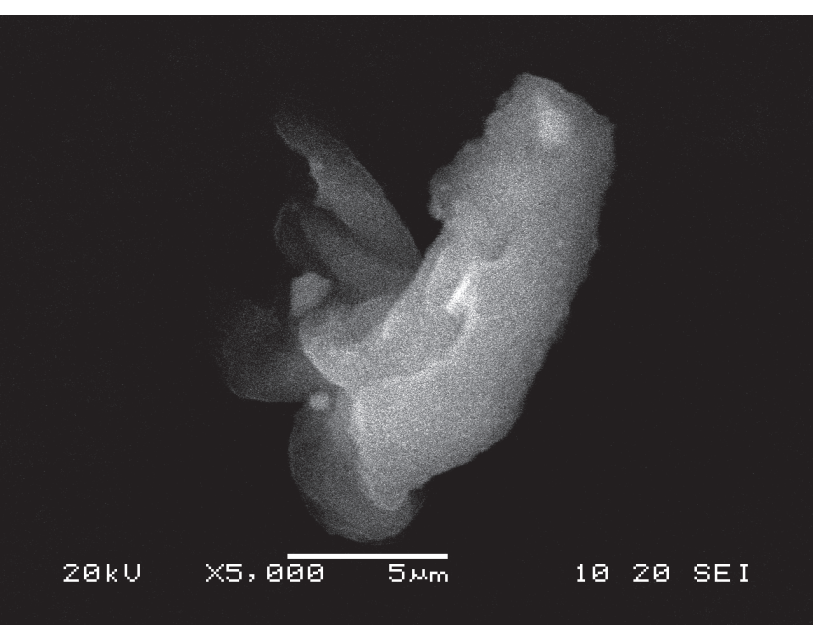

$d$

Рис. 3. СЭМ изображения планарных структур, образованных в результате полевого расслоения материала электрода: $a-\mathrm{Sb}, \times 8000,20$ кB; $b-\mathrm{Bi}, \times 5500,20$ кB; $c-\mathrm{InSb}, \times 4000,20$ кB; $d-\mathrm{SiC}, \times 5000,20$ кB

[Fig. 3. SEM images of planar structures formed as a result of field exfoliation of the electrode material: $a-\mathrm{Sb}, \times 8000$, $20 \mathrm{kV} ; b-\mathrm{Bi}, \times 5500,20 \mathrm{kV} ; c-\mathrm{InSb}, \times 4000,20 \mathrm{kV} ; d-\mathrm{SiC}, \times 5000,20 \mathrm{kV}]$

из-за высокой анизотропии. Ві формировал сферы, симметрично покрытые круглыми выступами, и небольшое количество отделившихся слоев из-за его низкой анизотропии. $\mathrm{SiC}$ не плавился или не испарялся при температуре разряда, а расслаивался, поэтому он производил плоские структуры.

\section{ВЫВОДЫ}

При воздействии импульсной плазмы на слоистые материалы наблюдается не только поверхностное плавление и испарение, приводящие к образованию хорошо известных сферических и фрактальных структур, но и расслоение с образованием мультислоев. Расслоение приводит к образованию сложных структур, имеющих признаки самоорганизации. При использовании барьерного разряда и исключении тепловой составляющей окисление $\mathrm{SiC}$ не происходит, и образуются мультислои. Ре- зультаты могут быть использованы при создании гибридных наноматериалов.

Работа выполнена при финансовой поддержке РФФИ (проект № 16-43-360281 p_a).

Выражаем благодарность Центру коллективного пользования научным оборудованием Воронежского государственного университета за активную поддержку работ молодых ученых.

\section{СПИСОК ЛИТЕРАТУРЫ}

1. Ares P., Aguilar-Galindo F., Rodriguez-SanMiguel D., et al. // Adv. Mater., 2016, № 28 (30), pp. 6332 6336. DOI: $10.1002 /$ adma.201602128

2. Gibaja C., Rodriguez-San-Miguel D., Ares P., et al. // Angew. Chem. Int. Ed., 2016, № 55 (46), pp. 14345-14349. DOI: 10.1002 /anie. 201605298

3. Jianping Ji, Xiufeng Song, Jizi Liu, et al. // Nature Communications, 2016, vol. 7, p. 13352. DOI: 10.1038/ ncomms 13352 


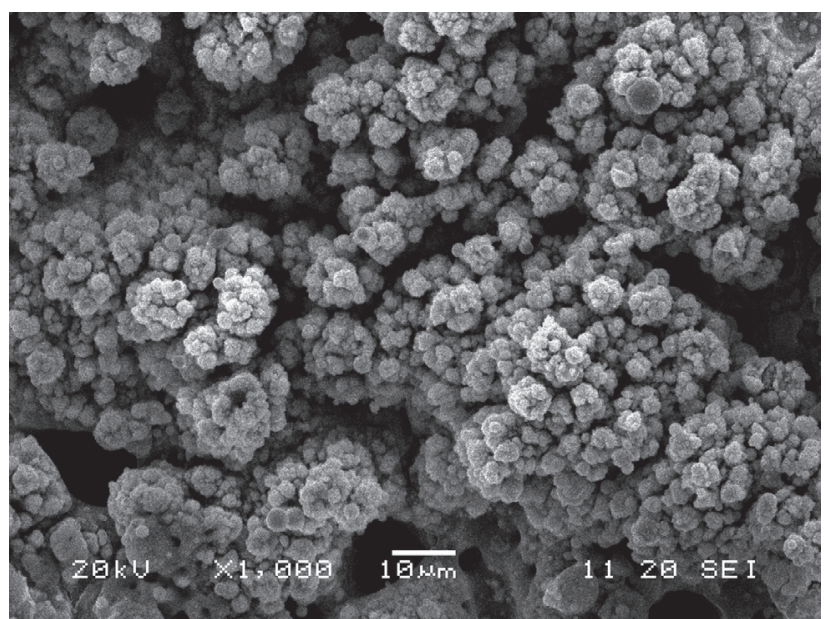

$a$

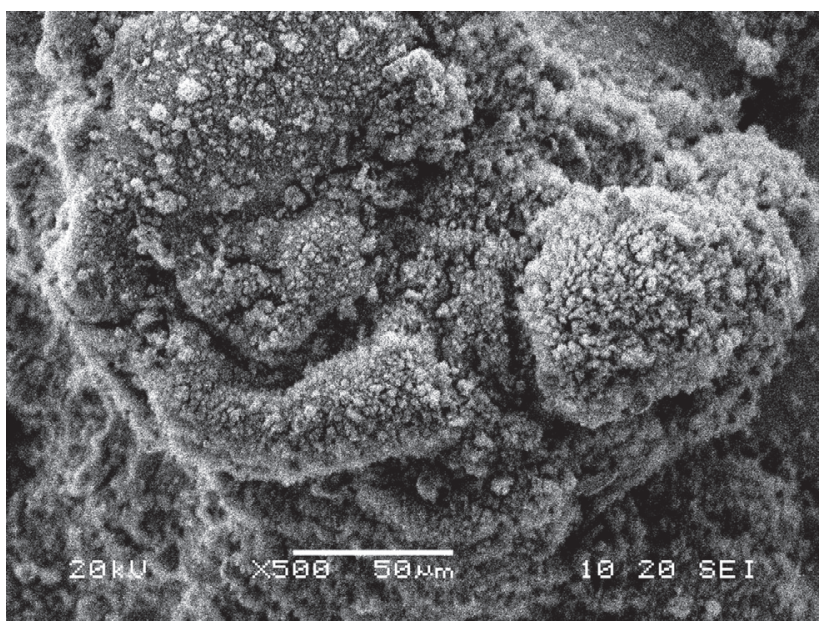

$b$

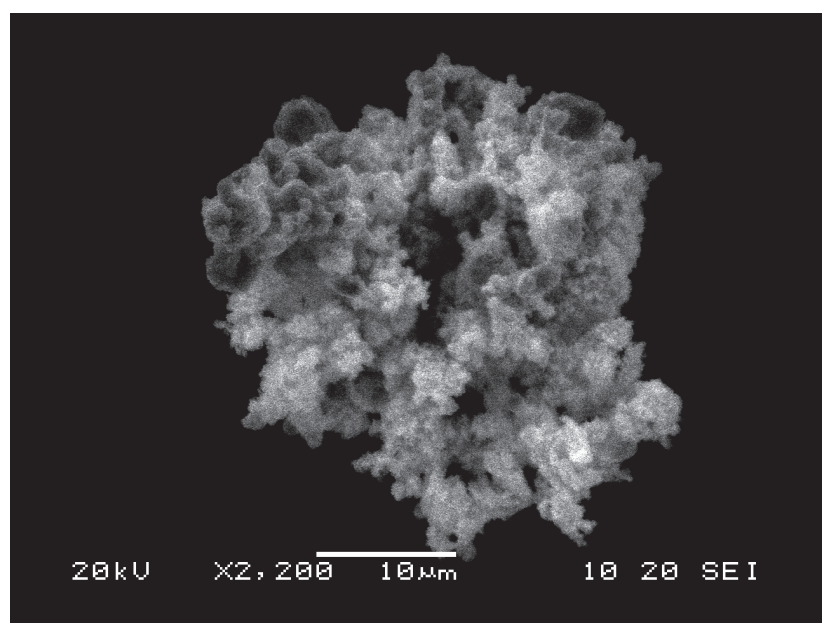

$c$

Рис. 4. СЭМ изображения фрактальных структур, образовавшихся при конденсации заряженных паров материала электрода на их поверхности: $a-\mathrm{Sb}, \times 1000,20$ кB; $b-\mathrm{Bi}, \times 500,20$ кB; $c-\mathrm{InSb}, \times 2200,20$ кB

[Fig. 4. SEM images of fractal structures formed during the condensation of charged vapor of electrode material on their surface: $a-\mathrm{Sb}, \times 1000,20 \mathrm{kV} ; b-\mathrm{Bi}, \times 500,20 \mathrm{kV} ; c-\mathrm{InSb}, \times 2200,20 \mathrm{kV}]$

4. Куликова Т. В., Тучин А. В., Аверин А. А., Тестов Д. А., Битюцкая Л. А., Бормонтов Е. Н. // ЖТФ, 2018, т. 88, вып. 7, с. 1025-1031.

5. Куликова Т. В., Битюцкая Л. А., Тучин А. В., Аверин А. А. // Перспективные материаль, 2017, № 3, c. $5-13$.

6. Куликова Т. В., Тучин А. В., Тестов Д. А., Битюцкая Л. А. // Конденсированные среды и межфазные гранищьь, 2017, т. 19, № 3, с. 368-375.

7. Maria E. Messing // Journal of Green Engineering, 2016, vol. 5, p. 83-96. DOI: 10.13052/jge1904-4720.5346

8. Ефимов А. А., Иванов В. В., Багазеев А. В., Бекетов И. В., Волков И. А., Щербинин С. В. // Письма в ЖТФ, 2013, т. 39, № 23, с. 51-57.

9. Арсенов П. В., Ефимов А. А., Мыльников Д. А., Лизунова А. А., Иванов В. В. // “Исследование процеессов получения аэрозольных наночастиц при импульсном газовом разряде между кремниевыми электродами", сборник трудов 59-й научной конференции МФТИ с международным участием, 21-26 ноября 2016 г, Москва - Долгопрудный - Жуковский, 2016, с. 49.

10. Lafont U., Simonin L., Tabrizi N. S., Schmidt-Ott A., Kelder E. M. // Journal of Nanoscience and Nanotechnology, 2009, vol. 9, pp. 2546-2552.

11. Лозанский Э. Д. // УФН, 1975, т. 117, № 3, c. 493-519.

12. Балданов Б. Б. // ЖТФ, 2011, т. 81, № 4, с. 135137.

13. Балданов Б. Б. Дисс. ... док. техн. наук. Томск, 2017,29 с.

14. Tabrizi N. S. Generation of Nanoparticles by Spark Discharge. Doctoral thesis, Tehran, 2009, p. 112.

15. Meuller B. O., Messing M. E., Engberg D. L. J., Jansson A. M., Johansson L. I. M., Norlén S. M., Tureson N., Deppert K. // Aerosol Science and Technology, 2012, pp. 1256-1270. DOI: 10.1080/02786826.2012.705448

16. Алейник Н. А. Плазменная медищина: Учеб. пособие. Томск, ТПУ, 2011, 40 с. 
17. Mariotti D., Sankaran R. M. // Journal of Physics D: Applied Physics, 2010, pp. 1-21. DOI: 10.1088/00223727/43/32/323001

18. Пискарев И. М., Иванова И. П., Трофимова С. В., Аристова Н. А. // Химия высоких энергий, 2012, т. 46, № 5, c. 406-411.
19. Swihart M. T. // Current Opinion in Colloid and Interface Science, 2003, vol. 8, pp. 127-133. DOI: 10.1016/ S1359-0294(03)00007-4

\title{
FORMATION OF SELF-ORGANIZED AND PLANAR STRUCTURES IN A MICROPLASMA OF A SPARK DISCHARGE
}

\author{
(c) 2018 I. I. Dolgih, D. V. Avdeev, T. V. Kulikova, L. A. Bitjuckaja \\ Voronezh State University, 1 Universitetskaya pl., 394018 Voronezh Russia \\ e-mail:dolgih_igor@yahoo.com
}

Received 24.04.2018

\begin{abstract}
We studied the action of the pulsed micro plasma on the layered materials with the different interlayer bond energy. The pulsed micro plasma was generated by the spark discharges between the two pieces of studied material in dry air at normal conditions in an open reactor. The $20 \mathrm{kV}$ spark discharges were generated with induction coil and had the duration from 10 to 20 us, controlled with an oscilloscope with a capacitive sensor. The generated particles were accumulated on the duct tape underneath the electrodes. Three types of particles were observed - droplets, fractals and planar structures. Droplets were produced by surface melting of the electrode material with the subsequent separation of a drop. The drops had different forms depending on the material. Sb produced spheres, $\mathrm{Bi}$ formed spheres covered symmetrically with round tips, InSb produced twisted structures. The planar structures were produced by the field exfoliation of the electrode material. Fractals were produced on the electrodes because of the circular evaporation and condensation of the material in pulsed plasma. The ratio of these three effects was determined by the degree of anisotropy of the processed material and by its melting point. Antimony produced many droplets, fractals and layers, because of its low melting point and high anisotropy. Bi produced droplets, fractals and a little amount of exfoliated layers because of its low anisotropy. $\mathrm{SiC}$ did not melt or evaporate at the temperature of the discharge but exfoliated, so it produced planar structures. The results may be used in the production of micro and nano particles needed to create hybrid materials.
\end{abstract}

Keywords: micro plasma, micro particles, layered precursors, spark discharge, self-organization.

DOI: https://doi.org/10.17308/kcmf.2018.20/525

\section{ACKNOWLEDGMENTS}

The reported study was supported by the Russian Foundation for Basic Research (project No. 16-43$360281 r_{-}$).

The research results were obtained with equipment of Voronezh State University Centre for Collective Use of Scientific Equipment.

\section{REFERENCES}

1. Ares P., Aguilar-Galindo F., Rodriguez-San-Miguel D., et al. Adv. Mater., 2016, no. 28 (30), pp. 6332-6336. DOI: $10.1002 /$ adma.201602128

2. Gibaja C., Rodriguez-San-Miguel D., Ares P., et al. Angew. Chem. Int. Ed., 2016, no. 55 (46), pp. 14345-14349. DOI: $10.1002 /$ anie. 201605298
3. Jianping Ji, Xiufeng Song, Jizi Liu, et al. Nature Communications, 2016, vol. 7, p. 13352. DOI: 10.1038/ ncomms 13352

4. Kulikova T. V., Tuchin A. V., Averin A. A., Testov D. A., Bityutskaya L. A., Bormontov E. N. Journal of Technical Physics, 2018, vol. 88, no. 7, pp. 1025-1031. (in Russ.)

5. Kulikova T. V., Bityutskaya L. A., Tuchin A. V., Averin A. A. Perspektivnye Materialy, 2017, no. 3, pp. 5-13. (in Russ.)

6. Kulikova T. V., Tuchin A. V., Testov D. A., Bitjuckaja L. A. Condensed Matter and Interfases, 2017, vol. 19, no. 3, pp. 368-375. Available at: http://www.kcmf.vsu.ru/ resources/t_19_3_2017_007.pdf (in Russ.)

7. Maria E. Messing Journal of Green Engineering, 2016, vol. 5, pp. 83-96. DOI: 10.13052/jge19044720.5346 
8. Efimov A. A., Ivanov V. V., Bagazeev A. V., Beketov I. V., Volkov I. A., Shherbinin S. V. Pis'ma v ZhTF, 2013, vol. 39, no. 23, pp. 51-57. (in Russ.)

9. Arsenov P. V., Efimov A. A., Myl'nikov D. A., Lizunova A. A., Ivanov V. V. “Issledovanie processov poluchenija ajerozol'nyh nanochastic pri impul'snom gazovom razrjade mezhdu kremnievymi jelektrodami" [Investigation of the processes of obtaining aerosol nanoparticles under a pulsed gas discharge between silicon electrodes], Proc. LIX Int. Conf., 21-26 November, 2016, Moscow - Dolgoprudny - Zhukovsky, 2016, p. 49.

10. Lafont U., Simonin L., Tabrizi N. S., Schmidt-Ott A., Kelder E. M. Journal of Nanoscience and Nanotechnology, 2009, vol. 9, pp. 2546-2552.

11. Lozanskij E. D. Physics-Uspekhi [Advances in Physical Sciences], 1975, vol. 117, no. 3, pp. 493-519. (in Russ.)

12. Baldanov B. B. Technical Physics, 2011, vol. 56, no. 4, pp. 564-566. DOI: https://doi.org/10.1134/ S1063784211040062
13. Baldanov B. B. Diss. doc. tech. nauk. Tomsk, 2017, $29 \mathrm{p}$.

14. Tabrizi N. S. Generation of Nanoparticles by Spark Discharge. Doctoral thesis, Tehran, 2009, p. 112.

15. Meuller B. O., Messing M. E., Engberg D. L. J., Jansson A. M., Johansson L. I. M., Norlén S. M., Tureson N., Deppert K. Aerosol Science and Technology, 2012, pp. 1256-1270. DOI: 10.1080/02786826.2012.705448

16. Aleinik N. A. Plazmennaja medicina: Uchebnoe posobie [Plasma Medicine: Educational allowance]. Tomsk, TPU Publ., 2011, 40 p. (in Russ.)

17. Mariotti D., Sankaran R. M. Journal of Physics D: Applied Physics, 2010, pp. 1-21. DOI:10.1088/00223727/43/32/323001

18. Piskarev I. M., Ivanova I. P., Trofimova S. V., Aristova N. A. Himija vysokih jenergij [High Energy Chemistry], 2012, vol. 46, no. 5, p. 343. DOI: 10.1134/S001814391 2050050

19. Swihart M. T. Current Opinion in Colloid and Interface Science, 2003, vol. 8, pp. 127-133. DOI: 10.1016/ S1359-0294(03)00007-4
Долгих Игорь Игоревич - аспирант кафедры физики полупроводников и микроэлектроники, Воронежский государственный университет; тел.: +7(908) 1468527, e-mail: dolgih_igor@yahoo.com

Авдеев Дмитрий Владимирович - студент, Воронежский государственный университет; тел.: +7(952)5515819, e-mail: avdoss@bk.ru

Куликова Татьяна Валентиновна - к. ф.-м. н., инженер кафедры физики полупроводников и микроэлектроники, Воронежский государственный университет; тел.: +7(908) 1445155, e-mail: kaimt@mail.ru

Битюикая Лариса Александровна - к. х. н., доцент кафедры физики полупроводников и микроэлектроники, Воронежский государственный университет; тел.: +7(473) 2208481, e-mail: me144@ phys.vsu.ru
Igor I. Dolgih - graduate student, Department of Physics of Semiconductors and Microelectronics, Voronezh State University; tel.: +7(908) 1468527, e-mail: dolgih_igor@yahoo.com

Dmitry V. Avdeev - student, Voronezh State University; tel.: +7(952)5515819, e-mail: avdoss@, bk.ru

Tatiana V. Kulikova - Cand. Sci. (Phys.Math.), Engineer of the Department of Physics of Semiconductors and Microelectronics, Voronezh State University; tel.: +7(908) 1445155, e-mail: kaimt@, mail.ru

Bityutskaya Larisa A. - Cand. Sci. (Chem.), Associate Professor, Department of Physics of Semiconductors and Microelectronics, Voronezh State University; tel.: +7(473) 2208481, e-mail: me144@ phys.vsu.ru 